\title{
Opinion
}

\section{Fertility Preservation in the Time of COVID-19}

\author{
Jan Tesarik \\ MARGen Clinic, Camino de Ronda 2, 18006 Granada, Spain \\ Address correspondence to Jan Tesarik, jtesarik@clinicamargen.com
}

Received 15 September 2020; Revised 1 February 2021; Accepted 23 February 2021

Copyright $@ 2021$ Jan Tesarik. This is an open access article distributed under the terms of the Creative Commons Attribution License, which permits unrestricted use, distribution, and reproduction in any medium, provided the original work is properly cited.

\begin{abstract}
Background. Fertility preservation has always been an important part of reproductive medicine. It has acquired a new dimension in the current period of the Coronavirus crisis. Objective. To review published data about the possible effects of COVID-19 on human reproductive function and propose possible solutions to be used when fertility of affected people is worsening. Methods. Search of scientific literature. Findings. The effects of COVID-19 on affected people's reproductive function in the medium and long term cannot be predicted with certainty. Fertility may also be affected by some treatment agents used against COVID-19. Fertility preservation in vivo, suitable in young persons with no apparent fertility problems, can be made with the use of nontoxic and generally fertility-friendly agents, such as melatonin and vitamin D. For older persons and those with already present fertility problems, in vitro fertility preservation, using gamete or embryo freezing, appears preferable. Finally, the same nontoxic agents recommended for in vivo fertility preservation should be given systematically to all pregnant women to protect them against infection during this critical period of life. Conclusion. Markers of reproductive function integrity should be evaluated in all people of reproductive age affected by COVID-19. According to the evolution of their values, and taking into account the overall condition of each patient, appropriate fertility preservation methods have to be chosen.
\end{abstract}

Keywords SARS-CoV-2; COVID-19; sperm quality; oocyte quality; anti-COVID treatment; gamete and embryo cryopreservation; in vivo fertility preservation

\section{Introduction}

Fertility preservation has been a significant concern of clinicians and scientists working in infertility diagnosis and treatment for several decades. Family planning, with the progressively increasing delay of motherhood to still more advanced female ages, was the leading cause of this concern. The recent outbreak of the COVID-19 pandemic has added a new dimension to this issue. Nevertheless, SARS-CoV-2, the coronavirus responsible for COVID-19 disease, is highly unlikely to enter mature human gametes [1], because the virus employs the angiotensin-converting enzyme 2 receptor (ACE2) for entry into human cells and this receptor is not present in mature human oocytes and spermatozoa [1].

However, ACE2 is abundant in testicular and ovarian cells, other than the gametes, that are important for appropriate support of gamete development and maturation. In the ovary, theca cells and granulosa cells can be infected. The former are involved in ovarian steroidogenesis, whereas the latter, in addition to participating in steroidogenesis, are also intimately involved in the regulation of oocyte growth and maturation. As to the testis, both Leydig cells, responsible for steroidogenesis, and Sertoli cells, responsible for the protection and nutrition of spermatogenic cells throughout their development, can be infected. The infection of these types of ovarian and testicular cells can affect negatively the development of gametes. This is particularly the testis case, where hormonal alterations in patients with COVID-19 could lead to the formation of antisperm antibodies and subsequent immunological infertility, an increase in the sperm DNA fragmentation, and inflammation leading to spermatogenesis failure [2,3]. However, it has to be pointed out that, though probable, all these complications remain hypothetical and need further studies to be confirmed. Nevertheless, although the precise impacts of COVID-19 on fertility are yet to be determined, the use of noninvasive, in vivo fertility protection measures could be recommended as a precautionary measure in people of reproductive age or wishing to conceive.

Recent studies have suggested, though not yet definitively confirmed, that the virus can impair both female [4] and male [5] fertility in the medium and long term. The latter study [5] reported significant seminiferous tubule injury, reduced Leydig cells, and mild lymphocytic infiltration/inflammation in postmortem examination of 12 deceased COVID-19 patients. These findings, namely that of lymphocytic infiltration/inflammation of the seminiferous tubules, suggest that, in addition to disturbed gonadal somatic cell function, local/systemic inflammation, caused by COVID-19 infection, could also impair male and female fertility.

Even before the outbreak of COVID-19, more than a half of persons ( $76 \%$ of women and $61 \%$ of men) seeking infertility treatment showed anxiety symptomatology [6], and these figures are likely to further increase because of 
the uncertainty of whether and how COVID-19 will also impair the fertility status of these people.

This Opinion article focuses on measures that can be taken for the protection of gametes from in vivo damage, applicable to young persons who are still healthy, cryostorage of gametes and embryos for already infected persons in the asymptomatic phase or those showing mild symptoms of the disease, avoidance of the use of therapeutic agents with known or suspected gametotoxic effects for persons in reproductive age, and the protection of pregnant women against the potential impacts of COVID-19 on pregnancy outcome.

\section{In vivo gamete protection}

Reducing the risk of contagion is the best way of protecting gametes in vivo. The use of conventional measures, such as face masks, respecting security distance between persons, frequent handwashing, or using disposable towels and tissues, should be most effective. However, these measures depend on everyone's goodwill, and recent experience is teaching us that this condition is not always met. In addition to these conventional measures, there is abundant circumstantial evidence suggesting that oral intake of melatonin (5-10 mg daily) provides an effective, though unspecific, protection against COVID-19 contagion [7,8], and if it has already occurred, prevents it from passing from asymptomatic and mild phases to severe and life-threatening ones $[9,10]$. Recent data strongly suggest that melatonin also has direct antiviral action by inhibiting growth factor receptors required for SARS-CoV-2 replication within the infected cells [11]. However, these suggestions still need to be confirmed by adequately powered randomized controlled trials (RCTs). There is no severe side-effect of melatonin, even at much higher doses than those recommended in this article. As a cautionary note, however, it has to be mentioned that, in some persons, melatonin can produce disturbed sleep, irritability and depression, even though these reactions are extremely rare. All these minor side-effects are completely reversible after stopping melatonin treatment.

In addition to melatonin, vitamin $\mathrm{D}$ has emerged recently as another unspecific but likely beneficial agent to combat COVID-19, reviewed in [12]. The combined use of vitamin D and melatonin to prevent and treat COVID-19 appears reasonable because the mode of action of each of these agents is complementary, the former enhancing the overall immune response to the pathogenic agent [12] and the latter modulating the initial innate (blind) response to an adaptative (specific) one [9,10]. Hopefully, these agents will prove efficient to be used for COVID-19 instead of other, antiviral agents, such as the repurposed drugs chloroquine, hydroxychloroquine, lopinavir/ritonavir, umifenovir, remdesivir, favipiravir, tocilizumab, most of which produce side-effects in different organs (such as gastrointestinal system, heart, liver, kidneys, central nervous system, and bone marrow), while others (interferon, ribavirin) interfere with gonadal function [13]. The contrary is true for vitamin $\mathrm{D}$ and melatonin. The former is beneficial for bone and muscle health [11]. The latter possesses several effects against various human pathologies, including infertility due to endometriosis and adenomyosis, prevention of different types of cancer, and preventing Alzheimer's disease [8]. Moreover, both agents can protect against other seasonal viral respiratory infections, such as common flu, that may develop simultaneously with COVID-19 and complicate further the clinical picture of the disease.

Given the above considerations, it is firmly recommendable to start using vitamin $\mathrm{D}$ and melatonin against COVID19 as soon as possible, without waiting for definitive confirmation of their efficiency through RCTs. Building up RCTs to test the efficiency of vitamin D and melatonin against COVID-19 may contradict the commonly accepted ethical rules, according to which, before an RCT can be conducted, there must be equipoise-genuine doubt about whether one course of action is better than another [14]. Accordingly, if the evidence, even the circumstantial one, suggests that patients in one arm of the study are more likely to benefit from enrollment than patients in the other arm, there are strong doubts about the corresponding RCTs' ethical justification [7]. Thus, in the case of vitamin $\mathrm{D}$ and melatonin as preventive and therapeutic agents against COVID-19, we should follow the same reasoning adopted by the World Health Organization (WHO) in the case of Ebola disease. In fact, given the gravity of the situation, WHO recommended starting experimental treatments of Ebola before obtaining definitive confirmatory data from RCTs [15]. This precedent should also be considered for COVID-19, and even more seriously because of the demonstrated lack of negative side-effects of the recommended agents. The circumstantial evidence in favor of melatonin is not worse than that available for any of the currently distributed anti-COVID-19 vaccines, most of which still have incompletely documented side-effect risks. Anyway, the immediate preventive use of melatonin and vitamin D should only be considered as a precautionary measure, given the fact that the impact of COVID-19 infection on fertility is yet to be determined and damage to the reproductive organs and gametes may be permanent. It should also be stressed in this context that the use of melatonin and vitamin $\mathrm{D}$ has no known negative impacts to fertility/reproductive function.

\section{Gamete and embryo cryopreservation}

Even though gametes' in vivo protection is a viable option in some cases, gamete or embryo cryopreservation is a better alternative in others. However, this option should not be recommended indiscriminately to all patients. Its 
adequacy should always be evaluated in the clinical context of the persons in question. As to sperm cryopreservation, it is an entirely safe, noninvasive, and inexpensive procedure. On the other hand, oocyte retrieval is a relatively invasive, stressful, and expensive process. Hence, it should only be taken into account in women already planned for in vitro fertilization, who are at risk of a significant oocyte quality decline due to the delay of treatment because of different kinds of problems related to the pandemic. A patienttailored approach and informed consent by the couple are needed to take this kind of decision.

In the first place, cryopreservation should be considered when other serious and pandemic-independent factors threatening fertility are also present. Due to the imprevisible evolution of the COVID-19 pandemic, a simple loss of time could significantly impact the patients' chances of pregnancy success. This applies above all to women of advanced age due to their likely reduced oocyte quantity and quality.

Sperm cryopreservation can also be recommended to men of reproductive age who are in an asymptomatic or mild-symptomatic phase of COVID-19 disease because of imprevisible long-term effects of the virus on their sperm production and quality. Unlike sperm, preventive oocyte cryopreservation would be much more complicated and expensive. It would also be burdened by security issues. It should thus be avoided except for rare situations where there are strong arguments in favor.

From a technical point of view, sperm and embryo cryopreservation gives excellent results. According to recent studies $[16,17]$, frozen embryo transfers perform better in single live birth than fresh embryo transfers in stimulated cycles, presumably because ovarian stimulation negatively affects endometrial receptivity. On the other hand, the outcomes of ICSI with cryopreserved oocytes are worse as compared with fresh oocytes [18], mainly because the incubation of oocytes with cryoprotectants depletes calcium ions from intracellular stores (endoplasmic reticulum and mitochondria), leading to insufficient calcium signaling in response to the fertilizing spermatozoon [19, 20]. It appears that this problem can be overcome by assisted oocyte activation [21], using calcium ionophores, or specific mechanical manipulation during ICSI [22,23], but this possibility remains to be confirmed.

\section{Protection of ongoing pregnancy}

In contrast to early alarming reports (not included in this article), based on limited numbers of cases, later and better-powered studies consider the transmission of COVID-19 infection from the mother to the child to be quite improbable [24,25]. Nevertheless, serious health problems, resulting from COVID-19, in the mother may have nonspecific adverse effects on pregnancy and parturition [24,25]. Here again, the use of nonspecific but nontoxic preventive measures, such as melatonin and vitamin $\mathrm{D}$, is of particular interest in pregnant women. In addition to its supposed effect against COVID-19 (see above), melatonin has a well-known beneficial impact on pregnancy in healthy women, too [26].

\section{Integrative view and conclusions}

As discussed above, there are different methods potentially useful for fertility preservation facing the actual Coronavirus crisis. The use of each of these methods and the choice of the adequate one will depend on every person's current situation in question. In vivo-acting agents, such as melatonin and vitamin D, may be sufficient in healthy persons with no fertility problems. However, gamete or embryo freezing will be preferable for older persons, mainly women, and those with pre-existing fertility problems. Finally, melatonin and vitamin D are highly recommendable for all pregnant women to avoid infection during this particular period of life. In conclusion, even though the potential impacts of COVID-19 on fertility remain unconfirmed and speculative, the possible damage to gametes/gonads can be permanent. Hence, as a precautionary measure, we should be considering fertility preservation even before the precise impacts are discovered.

Conflict of interest The author declares that he has no conflict of interest.

\section{References}

[1] J. Tesarik, After corona: there is life after the pandemic, Reprod Biomed Online, 40 (2020), 760-762.

[2] L. Ma, W. Xie, D. Li, L. Shi, Y. Mao, Y. Xiong, et al., Effect of SARS-CoV-2 infection upon male gonadal function: A single center-based study, preprint.

[3] A. Haghpanah, F. Masjedi, S. Alborzi, A. Hosseinpour, A. Dehghani, L. Malekmakan, et al., Potential mechanisms of SARS-CoV-2 action on male gonadal function and fertility: Current status and future prospects, Andrologia, 53 (2021), e13883.

[4] J. Yan, R.-Q. Li, H.-R. Wang, H.-R. Chen, Y.-B. Liu, Y. Gao, et al., Potential influence of COVID-19/ACE2 on the female reproductive system, Mol Hum Reprod, 26 (2020), 367-373.

[5] M. Yang, S. Chen, B. Huang, J. M. Zhong, H. Su, Y. J. Chen, et al., Pathological findings in the testes of COVID-19 patients: clinical implications, Eur Urol Focus, 6 (2020), 1124-1129.

[6] L. A. Pasch, S. R. Holley, M. E. Bleil, D. Shehab, P. P. Katz, and N. E. Adler, Addressing the needs of fertility treatment patients and their partners: are they informed of and do they receive mental health services?, Fertil Steril, 106 (2016), 209-215.e2.

[7] R. J. Reiter, P. Abreu-Gonzalez, P. E. Marik, and A. DominguezRodriguez, Therapeutic algorithm for use of melatonin in patients with COVID-19, Front Med (Lausanne), 7 (2020), 226.

[8] J. Tesarik, Melatonin to reduce death toll due to COVID-19: from innate to adaptive immune response, Glob J Med Res, 20 (2020).

[9] R. Zhang, X. Wang, L. Ni, X. Di, B. Ma, S. Niu, et al., COVID19: Melatonin as a potential adjuvant treatment, Life Sci, 250 (2020), 117583.

[10] D. X. Tan and R. Hardeland, Potential utility of melatonin in deadly infectious diseases related to the overreaction of innate immune response and destructive inflammation: focus on COVID-19, Melatonin Res, 3 (2020), 120-143. 
[11] J. Tesarik, Melatonin attenuates growth factor receptor signaling required for SARS-CoV-2 replication, Melatonin Res, 3 (2020), 534-537.

[12] A. R. Martineau and N. G. Forouhi, Vitamin D for COVID-19: a case to answer?, Lancet Diabetes Endocrinol, 8 (2020), 735-736.

[13] J. M. Sanders, M. L. Monogue, T. Z. Jodlowski, and J. B. Cutrell, Pharmacologic treatments for coronavirus disease 2019 (COVID-19): A review, JAMA, 323 (2020), 1824-1836.

[14] S. W. Duffy, Interpretation of the breast screening trials: a commentary on the recent paper by Gøtzsche and Olsen, Breast, 10 (2001), 209-212.

[15] C. Adebamowo, O. Bah-Sow, F. Binka, R. Bruzzone, A. Caplan, J. F. Delfraissy, et al., Randomised controlled trials for Ebola: practical and ethical issues, Lancet, 384 (2014), 1423-1424.

[16] D. Wei, J. Y. Liu, Y. Sun, Y. Shi, B. Zhang, J. Q. Liu, et al., Frozen versus fresh single blastocyst transfer in ovulatory women: a multicentre, randomised controlled trial, Lancet, 393 (2019), 1310-1318.

[17] M. Roque, T. Haahr, S. Geber, S. C. Esteves, and P. Humaidan, Fresh versus elective frozen embryo transfer in IVF/ICSI cycles: a systematic review and meta-analysis of reproductive outcomes, Hum Reprod Update, 25 (2019), 2-14.

[18] V. A. Kushnir, S. K. Darmon, D. H. Barad, and N. Gleicher, New national outcome data on fresh versus cryopreserved donor oocytes, J Ovarian Res, 11 (2018), 2.

[19] D. Nikiforaki, F. Vanden Meerschaut, C. Qian, I. De Croo, Y. Lu, T. Deroo, et al., Oocyte cryopreservation and in vitro culture affect calcium signalling during human fertilization, Hum Reprod, 29 (2014), 29-40.

[20] D. Bonte, V. Thys, P. De Sutter, A. Boel, L. Leybaert, and B. Heindryckx, Vitrification negatively affects the $\mathrm{Ca}^{2+}$-releasing and activation potential of mouse oocytes, but vitrified oocytes are potentially useful for diagnostic purposes, Reprod Biomed Online, 40 (2020), 13-25.

[21] J. Tesarik, Can the negative effects of vitrification on oocyte developmental competence be mitigated?, Reprod Biomed Online, 41 (2020), 349.

[22] A. V. Rybouchkin, F. Van der Straeten, J. Quatacker, P. De Sutter, and M. Dhont, Fertilization and pregnancy after assisted oocyte activation and intracytoplasmic sperm injection in a case of round-headed sperm associated with deficient oocyte activation capacity, Fertil Steril, 68 (1997), 1144-1147.

[23] J. Tesarik, L. Rienzi, F. Ubaldi, C. Mendoza, and E. Greco, Use of a modified intracytoplasmic sperm injection technique to overcome sperm-borne and oocyte-borne oocyte activation failures, Fertil Steril, 78 (2002), 619-624.

[24] L. Zeng, S. Xia, W. Yuan, K. Yan, F. Xiao, J. Shao, et al., Neonatal early-onset infection with SARS-CoV-2 in 33 neonates born to mothers with COVID-19 in Wuhan, China, JAMA Pediatr, 174 (2020), 722-725.

[25] A. Khalil, E. Kalafat, C. Benlioglu, P. O’Brien, E. Morris, T. Draycott, et al., SARS-CoV-2 infection in pregnancy: $A$ systematic review and meta-analysis of clinical features and pregnancy outcomes, EClinicalMedicine, 25 (2020), 100446.

[26] L. G. A. Chuffa, L. A. Lupi, M. S. Cucielo, H. S. Silveira, R. J. Reiter, and F. R. F. Seiva, Melatonin promotes uterine and placental health: potential molecular mechanisms, Int J Mol Sci, 21 (2019), 300 